\title{
ACCELERATION AND GENERALIZATION OF SOME INFINITE SERIES
}

\author{
Dr. Gunjan A. Ranabhatt
}

Government Engineering College, Bhavnagar, Gujarat, India

Abstract
In this paper, we give new series representation for well-known mathematical constant such as $\pi$, Catalan Constant and
alternating series of the $K(a, b ; c, d)[4]$. Moreover, give new series representation Lerch Zeta function and its
generalizations H-Lerch Zeta function and G-LerchZeta function. We also provide the convergence of series and some
special case of it. Moreover, its relations with other mathematical functions like Riemann Zeta function, Poly-logarithm
function, Dirichlet beta function etc. All the series we have found provide quite useful analytic approximation.
In addition, it is possible apply the same strategy to other mathematical functions.
KEYWORDS: Generalization, Infinite Series

Received: May 06, 2020; Accepted: May 26, 2020; Published: Aug 19, 2020; Paper Id.: IJMPERDJUN2020807

\section{INTRODUCTION}

In this paper, we deal with the problem of improving the convergence of slowly convergent series where a large number of terms are need to reach desired accuracy. This problem has many applications in physical problems. At this point, we know that perturbation theory often gives series, which converge very slowly or do not converge at all.

Therefore, to solve the problem we propose a new method to accelerate some class of mathematical series, which does not rely on perturbative approach, that is an expansion in some small natural parameter. The method works by introducing an artificial dependence in the formulas upon an arbitrary parameter, and then by devising an expansion, which can be optimizes give faster rates of convergence.

Here, we will show that such a technique can be used to obtain exponentially convergent series for some mathematical function. A proof of convergence of the method is also providing. In this paper, we denote the sum of the series $\sum_{n=1}^{\infty}\left(\frac{a}{a n-b}-\frac{c}{c n-d}\right)$ of [4] by $K(a, b ; c, d)$.

As we know, many mathematical constants can be express infinite sums. In many cases, such a series converge very slowly and huge number of terms have to calculate before reaching desired precision.

First of all, we consider the series,

$$
S=8 \sum_{n=1}^{\infty}\left[\frac{1}{8 n-6}-\frac{1}{8 n-2}\right]
$$

The above series in equation (1) converges very slowly to $K(8,6 ; 8,2)=\pi$ using [4].

Flajolet and Vardi [1] have shown it is possible to convert series such as in above equation (1) into rapidly converging ones. Here, we generalize the method of Flajolet and Vardi. We introduce an arbitrary parameter in the 
series. Such a parameter, then tuned to accelerate the convergence of the series it-self by using Principle of Minimal Sensitivity (PMS) [8]. We can see it in our following results.

\section{Theorem 1:}

The series

$$
S=8 \sum_{m=1}^{\infty}\left(\frac{1}{1+\lambda}\right)^{m+1} \sum_{k=1}^{m}\left(\begin{array}{l}
m \\
k
\end{array}\right) \lambda^{m-k} \frac{6^{k}-2^{k}}{8^{k+1}} \zeta(k+1)
$$

Converge to $\pi$ for $\lambda>-1 / 2$, with $\lambda$ real.

\section{Proof:}

We can rewrite the series of equation (1) in the equivalent form, $S=\sum_{n=1}^{\infty} \frac{1}{n} \frac{1}{1+\lambda}\left(\frac{1}{\left.1-\frac{6 / 8 n+\lambda}{1+\lambda}-\frac{1}{1-\frac{2 / 8 n}{1+\lambda}}\right)}\right.$

where $\lambda \neq-1$ is an arbitrary parameter.

Here, provided that $\left|\frac{6 / 8 n+\lambda}{1+\lambda}\right|<1$ and $\left|\frac{2 / 8 n+\lambda}{1+\lambda}\right|<1$ for all $n \geq 1$.

This implies, $\lambda>-1$ for all $n \geq 1$.

Therefore, we can expand equation (2) as follows for $\lambda>-1$,

$$
S=8 \sum_{n=1}^{\infty} \frac{1}{8 n} \sum_{m=1}^{\infty}\left(\frac{1}{1+\lambda}\right)^{m+1} \sum_{k=1}^{m}\left(\begin{array}{l}
m \\
k
\end{array}\right) \lambda^{m-k}\left[\left(\frac{6}{8 n}\right)^{k}-\left(\frac{2}{8 n}\right)^{k}\right]
$$

As the above series (3) in $m$ and $n$ contains only positive terms, we can perform the series over $n$ and obtain the following result,

$$
S=8 \sum_{m=1}^{\infty}\left(\frac{1}{1+\lambda}\right)^{m+1} \sum_{k=1}^{m}\left(\begin{array}{l}
m \\
k
\end{array}\right) \lambda^{m-k} \frac{6^{k}-2^{k}}{8^{k+1}} \zeta(k+1)
$$

Now, we can write our series in equation (1) as

$$
S=8 \sum_{n=1}^{\infty}\left[\frac{1}{8 n-6}-\frac{1}{8 n-2}\right]=4 \sum_{n=1}^{\infty}\left[\frac{1}{4 n-3}-\frac{1}{4 n-1}\right]
$$

But in Vardi's book [[10], p. 159] gives an example Gregory's series for $\pi$,

$$
\frac{\pi}{4}=1-\frac{1}{3}+\frac{1}{5}-\frac{1}{7}+\ldots . .=\sum_{n=1}^{\infty}\left[\frac{1}{4 n-3}-\frac{1}{4 n-1}\right]
$$

However, the rearrangement of the above series gives following result [10], p. 159. 


$$
\pi=\sum_{m=1}^{\infty} \frac{3^{m}-1}{4^{m}} \zeta(m+1)
$$

\section{Remark 2}

The series of equation (2) for $\lambda=0$ coincides with the result of Flajolet and Vardi [1],

$$
S^{(F V)}=\sum_{m=1}^{\infty} \frac{3^{m}-1}{4^{m}} \zeta(m+1)
$$

Moreover, the series in above equation (5) converges to $\pi$.

Here, we observe that equation (2) define a family of series converging to $\pi$ with $\lambda>-1$. Clearly, the dependence upon $\lambda$ in equation (2) is artificial and shows up when a finite number of the terms is consider. However, we know that the series in equation (4) is the equivalent form of series in equation (2). Therefore, we can set $S_{N}(\lambda)$ to be the partial series of equation (4). Then the dependence upon $\lambda$ in $S_{N}(\lambda)$ disappears in the $\lim \quad N \rightarrow \infty$.

For, fixed $N$ we evaluate the partial sum at the point where,

$$
\begin{aligned}
& \frac{d}{d \lambda}\left(S_{N}(\lambda)\right)=0 \\
& \text { where } S_{N}(\lambda)=8 \sum_{m=1}^{N}\left(\frac{1}{1+\lambda}\right)^{m+1} \sum_{k=1}^{m}\left(\begin{array}{l}
m \\
k
\end{array}\right) \lambda^{m-k} \frac{6^{k}-2^{k}}{8^{k+1}} \zeta(k+1)
\end{aligned}
$$

since there the expression is less sensitive to change of the arbitrary parameter $\lambda$, a property which shares with whole series (4). This called Principle of Minimal Sensitivity (PMS). This provides an equation, which ones solved at the given order, provide an optimal value of $\lambda$ fir a fixed partial $\operatorname{sum} S_{N}(\lambda)$.

$$
\begin{aligned}
& \text { For } N=2, \text { we obtain } S_{2}(\lambda) \\
& \lambda_{1}=\frac{-3}{\pi^{2}} \zeta(3) \approx-0.365381>-1
\end{aligned}
$$

\section{Remark 3:}

The series of equation (4) converges geometrically to $\pi$. We can estimate the rate of convergence by approximating the $\mathrm{m}^{\text {th }}$ term in the series with

$$
S_{m}=\left(\frac{1}{1+\lambda}\right)^{m+1} \sum_{k=1}^{m}\left(\begin{array}{l}
m \\
k
\end{array}\right) \lambda^{m-k} \frac{6^{k}-2^{k}}{8^{k}}
$$

Therefore,

$$
S_{m}=\frac{1}{(1+\lambda)^{m+1}}\left[(\lambda+6 / 8)^{m}-(\lambda+2 / 8)^{n}\right] \approx m\left(\frac{\lambda+6 / 8}{1+\lambda}\right)^{m}
$$


Now, using the PMS value if equation (6) we obtain $S_{m} \approx 1.65^{-m}$. This improves the rate $S_{m} \approx 1.33^{-m}$ of the series of Flajolet and Vardi (5).

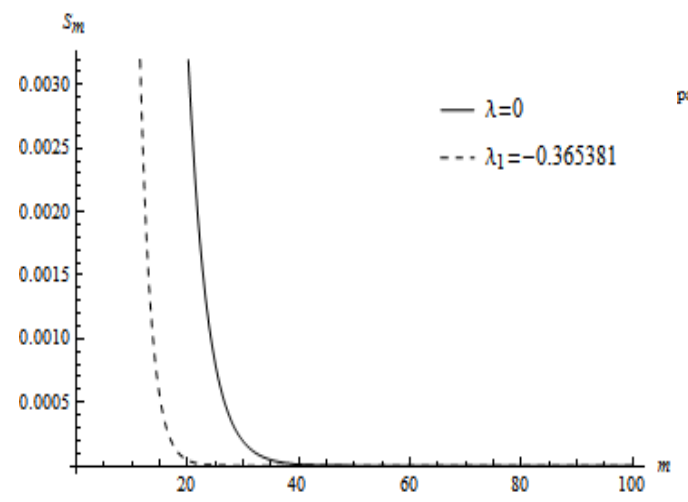

Figure 1

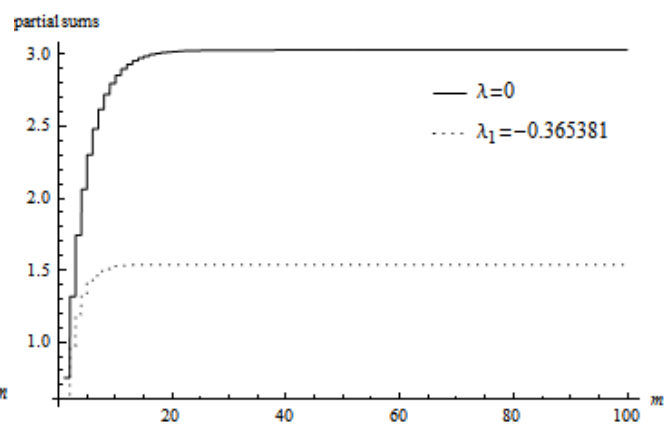

Figure 2

In figure 1 we display first 100 terms of $\mathrm{m}^{\text {th }}$ term $S_{m}$ when $S_{m} \approx 1.33^{-m}$ for $\lambda=0$ and $S_{m} \approx 1.65^{-m}$ for $\lambda_{1}=-0.365381$. Then, in figure 2, we plot partial sums of equation (4) up to 100 terms for $\lambda_{\text {and }} \lambda_{1}$. In above Figure the vertical lines turn out that $\lambda_{1}$ is a very good approximation to the minimum of partial sum even for large values of terms.

Next, in figure 3 we plot the error $\delta$ obtained in each terms up to 100 terms of equation (4) with $\lambda$ given by equation (7) and by using formula of Flajolet and Vardi, equation(6). Moreover, in last figure 4, we display the error $\delta$ obtained in each partial sums up to100 terms of equation (4) with $\lambda$ given by equation (7) and by using formula of Flajolet and Vardi, equation (6). Our series converges exponentially more rapidly than series in equation (6).

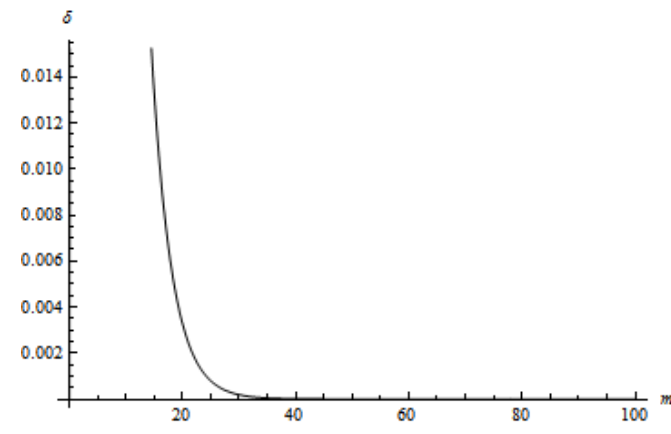

Figure 3

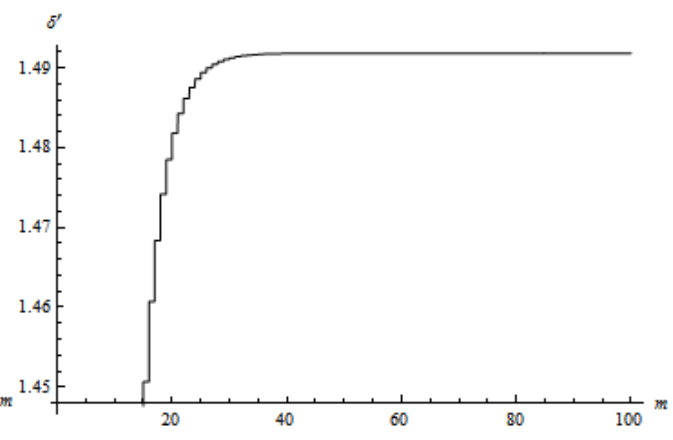

Figure 4

Now, we consider another series as follows:

$$
S=\sum_{n=1}^{\infty}\left[\frac{1}{(8 n-6)^{2}}-\frac{1}{(8 n-2)^{2}}\right]
$$

Moreover, the above series in equation (8) slowlyconvergesto $\frac{G}{4}$, where $G$ is the Catalan constant $G \approx 0.9159656$. 


\section{Theorem 4}

The series defined as

$$
S=\sum_{m=1}^{\infty}\left(\frac{1}{1+\lambda}\right)^{m+1} \sum_{k=1}^{m}\left(\begin{array}{l}
m \\
k
\end{array}\right) \lambda^{m-k} \frac{6^{k-1}-2^{k-1}}{8^{k}} k \zeta(k+1)
$$

Converges to $G / 4$, where $G$ is the Catalan Constant, $G \approx 0.9159656$ for any $\lambda>-1$, with $\lambda$ real.

\section{Proof:}

We can rewrite equation (8) as follows:

$S=\lim _{a \rightarrow 0} \frac{d}{d a} \bar{S}(a)$

where $\bar{S}(a)=\sum_{n=1}^{\infty}\left[\frac{1}{(8 n-6-a)}-\frac{1}{(8 n-2-a)}\right]$

Here, note that the above series $\bar{S}(a)$ converges uniformly in $a$. Therefore, we can differentiate term by term.

Then, by applying Theorem 1 to, $\bar{S}(a)$ we get

$\bar{S}(a)=\sum_{m=1}^{\infty} \sum_{k=1}^{m}\left(\frac{1}{1+\lambda}\right)^{m+1}\left(\begin{array}{l}m \\ k\end{array}\right) \lambda^{m-k} \frac{(6+a)^{k}-(2+a)^{k}}{8^{k}} \zeta(k+1)$

As the above series (10) converges uniformly in $a$, we have

$$
\frac{d}{d a} \bar{S}(a)=\sum_{m=1}^{\infty} \sum_{k=1}^{m}\left(\frac{1}{1+\lambda}\right)^{m+1}\left(\begin{array}{l}
m \\
k
\end{array}\right) \lambda^{m-k} \frac{(6+a)^{k-1}-(2+a)^{k-1}}{8^{k}} k \zeta(k+1)
$$

Therefore, form equation (10) and (12) we get our desired series as in equation (9).

Here, note that the series of Theorem 4 reduces to the formula for $\lambda=0$ as

$$
S^{(F V)}=\sum_{m=1}^{\infty}\left(\frac{3^{m-1}-1}{4^{m+1}}\right) m \zeta(m+1)
$$

Next, we generalize Theorem 1 for any integer $a>3$,

$$
S=a \sum_{n=1}^{\infty}\left[\frac{1}{a n-3}-\frac{1}{a n-1}\right]
$$

In addition, the above series in equation (14) converges to $K(a, 3 ; a, 1)$ of [4].

In addition, the parametric representation of above series (14) obtained in next theorem.

\section{Theorem 5:}

The series 
$S=a \sum_{m=1}^{\infty} \sum_{k=1}^{m}\left(\frac{1}{1+\lambda}\right)^{m+1}\left(\begin{array}{l}m \\ k\end{array}\right) \lambda^{m-k} \frac{3^{k}-1}{a^{k+1}} \zeta(k+1)$

Converge to $K(a, 3 ; a, 1)$ for $\lambda>-1 / 2$, with $\lambda$ real.

We can prove this theorem similar way as above theorem 1. Thenafter, in next remark we will see a special case of the series given by (15).

\section{Remark 4}

For $\lambda=0$, the above Theorem 5 reduces to the series

$$
S=\sum_{m=1}^{\infty} \frac{3^{m}-1}{a^{m}} \zeta(m+1)
$$

which is convergent for $a>3$.

For any integers $a, b$ and $c$ with conditions $a>b, a>c$ and $b \neq c$, we can generalize the series in (14) as

$$
S=a \sum_{n=1}^{\infty}\left[\frac{1}{a n-b}-\frac{1}{a n-c}\right]
$$

Moreover, the above series in equation (17) converges to $K(a, b ; a, c)$ of [4].

Therefore, in next theorem gives generalization of theorem 5.

\section{Theorem 6}

The series

$$
S=a \sum_{m=1}^{\infty} \sum_{k=1}^{m}\left(\frac{1}{1+\lambda}\right)^{m+1}\left(\begin{array}{l}
m \\
k
\end{array}\right) \lambda^{m-k} \frac{b^{k}-c^{k}}{a^{k+1}} \zeta(k+1)
$$

Converge to $K(a, b ; a, c)$ for $\lambda>-1 / 2$, with $\lambda$ real.

\section{Remark 5}

For $\lambda=0$, the above Theorem 6 reduces to the series

$$
S=\sum_{m=1}^{\infty} \frac{b^{m}-c^{m}}{a^{m}} \zeta(m+1)
$$

which is convergent for any integers $a, b$ and $c$ with conditions $a>b, a>c$ and $b \neq c$.

\section{Remark 6:}

Here, note that the Theorem 4 can generalized to sums of the form 


$$
S_{m}=\sum_{n=1}^{\infty}\left[\frac{1}{(a n-b)^{m}}-\frac{1}{(a n-c)^{m}}\right]
$$

for any integers $a, b$ and $c$ with conditions $a>b, a>c$ and $b \neq c$. Any integers $a, b$ and $c$ with conditions $a>b, a>c$ and $b \neq c$.

Now, we apply the same strategy outlined above to the calculation of Lerch zeta function $\Phi(z, s, x)$ is defined on p. 121 of [9] as follows:

$$
\Phi(z, s, x)=\sum_{n=0}^{\infty} \frac{z^{n}}{(n+x)^{s}}
$$

where $0<x \leq 1$ and $\operatorname{Re}(s)>1$

We have $\frac{e^{-y(x-1)}}{e^{y}-z}=\sum_{n=01}^{\infty} z^{n} e^{-y(x+n)}$

And $\quad \int_{0}^{\infty} e^{-y(x+n)} y^{s-1} d y=\frac{1}{(x+n)^{s}} \int_{0}^{\infty} e^{-t} t^{s-1} d t=\frac{\Gamma(s)}{(x+n)^{s}}$

Moreover, we have the integral representation in [9] at p. 121

$$
\Phi(z, \quad s, \quad x)=\frac{1}{\Gamma(s)} \int_{0}^{\infty} \frac{y^{s-1} e^{-y(x-1)}}{e^{y}-z} d y
$$

\section{Theorem 7}

A series for the Lerch zeta function, which is valid for $0<x \leq 1, \operatorname{Re}(s)>1$ and $\lambda>-\frac{1}{2}$ given by

$$
\Phi(z, \quad s, \quad x)=\sum_{m=0}^{\infty} \sum_{j=0}^{m}\left(\begin{array}{c}
m \\
j
\end{array}\right) \frac{\lambda^{m-j} \cdot z^{j}}{(1+\lambda)^{m+1}} \frac{1}{(x+j)^{s}}
$$

\section{Proof:}

We use the integral representation (22)

$$
\Phi(z, s, x)=\frac{1}{\Gamma(s)} \int_{0}^{\infty} \frac{y^{s-1} e^{-y(x-1)}}{e^{y}-z} d y
$$

Now, take change of variable $t=e^{-y}$ in above integral and introducing arbitrary parameter $\lambda$ by hand in equation we obtain

$$
\Phi(z, \quad s, \quad x)=\frac{1}{\Gamma(s)}\left(\frac{1}{1+\lambda}\right) \int_{0}^{1} \frac{\log ^{s-1}\left(\frac{1}{t}\right) \cdot t^{x-1}}{1-\frac{z t+\lambda}{1+\lambda}} d t
$$


Here, the condition $\left|\frac{z t+\lambda}{1+\lambda}\right|<1$ fulfilled uniformly for all $t \in[0,1]$ provided that $|z t|<1$ and $\lambda>-\frac{1}{2}$. In this case, we can expand the denominator in powers of $\left(\frac{z t+\lambda}{1+\lambda}\right)$ and obtain

$$
\Phi(z, \quad s, \quad x)=\frac{1}{\Gamma(s)} \sum_{m=0}^{\infty} \sum_{j=0}^{m}\left(\begin{array}{c}
m \\
j
\end{array}\right) \frac{\lambda^{m-j} \cdot z^{j}}{(1+\lambda)^{m+1}} \int_{0}^{1} \log { }^{s-1}\left(\frac{1}{t}\right) \cdot t^{x+j-1} d t
$$

Again, take change of variable $t=e^{-q}$ in above equation (25) we obtain

$$
\Phi(z, \quad s, \quad x)=\frac{1}{\Gamma(s)} \sum_{m=0}^{\infty} \sum_{j=0}^{m}\left(\begin{array}{c}
m \\
j
\end{array}\right) \frac{\lambda^{m-j} \cdot z^{j}}{(1+\lambda)^{m+1}} \int_{0}^{\infty} e^{-q(x+j)} \cdot q^{s-1} d q
$$

Now using the result $\frac{\Gamma(n)}{a^{n}}=\int_{0}^{\infty} e^{-a t} t^{n-1} d t$ of gamma function in (26), we get the desired result.

Now, we discuss the some properties of above series (23).The series (23) is independent of the arbitrary parameter $\lambda$, although the arbitrary parameter $\lambda$ appears explicitly in the series expression. This causes because the series in (21) is independent of $\lambda$ and in above theorem it has been just proved that the series in (23) converges to series in (21) provided that $|z t|<1$ and $\lambda>-\frac{1}{2}$. It means, we can say that the equation (23) gives the family of series, each corresponding to different value of $\lambda$ and each series in (23) converging to the same series (21).

Now, consider the partial sum

$$
\Phi^{N}(z, s, x)=\sum_{m=0}^{N} \sum_{j=0}^{m}\left(\begin{array}{c}
m \\
j
\end{array}\right) \frac{\lambda^{m-j} \cdot z^{j}}{(1+\lambda)^{m+1}} \frac{1}{(x+j)^{s}}
$$

The above series can obtain by restricting the infinite sum to the first $N+1$ terms. Clearly, the partial sum $\Phi^{N}(z, s, x)$ depends upon $\lambda$ as we are neglecting infinite numbers of terms. We can use this feature to our benefit and fix $\lambda$, therefore the convergence rate is maximal.

To obtain the proper values of $\lambda$, apply PMS to the equation (27), so we have

$$
\frac{d}{d \lambda} \Phi^{N}(z, s, x)=0
$$

For the lowest order, this corresponds to selecting $N=1$ we obtain the values

$$
\lambda_{P M S}^{1}=-\frac{1}{2}\left(1+\frac{z \cdot x^{s}}{(1+x)^{s}}\right)
$$

Then, for $s=2,3,4$, and fix value of $x$ we can find $\lambda_{P M S}^{1}$. And using the same strategy we can obtain 
higher order $\lambda_{P M S}$. By using these values we can obtain graph and see that the rate of convergence of the series greatly improved by applying PMS. Next, we see some special cases of above theorem for different values of $x, \lambda$ and $z$.

\section{Corollary 8}

Taking $x=\frac{b}{a}$ in the series (23), where $a$ and $b$ are positive integers with $b<a$. Then, for $\lambda>0$ the series (23) expressed as

$$
a^{s} \cdot \Phi(z, \quad s, \quad x)=\sum_{m=0}^{\infty} \sum_{j=0}^{m}\left(\begin{array}{c}
m \\
j
\end{array}\right) \frac{\lambda^{m-j} \cdot z^{j}}{(1+\lambda)^{m+1}} \frac{1}{(a j+b)^{s}}
$$

In next corollary, we will see another special case for $\lambda=1$ of series (23).

\section{Corollary 9}

Let $\lambda=1$ in the series $(23)$, then

$$
\Phi(z, s, x)=\sum_{m=0}^{\infty} \sum_{j=0}^{m}\left(\begin{array}{c}
m \\
j
\end{array}\right) \frac{z^{j}}{2^{m+1}} \frac{1}{(x+j)^{s}}
$$

Here, we note that in the definition of Lerch zeta function $\Phi(z, s, x)$ if we take $z=1$ then it is Hurwitz Zeta function $\zeta(s, x)$. We use this fact in next corollary.

\section{Corollary 10:}

Let $z=\lambda=1$ in the series (23), then we have

$$
\Phi(1, \quad s, x)=\zeta(s, x)=\sum_{m=0}^{\infty} \sum_{j=0}^{m}\left(\begin{array}{c}
m \\
j
\end{array}\right) \frac{1}{2^{m+1}(x+j)^{s}}
$$

Now, we generalize the Lerch zeta function as $H$ - Lerch zeta function, which defined as follows:

\section{Definition 11}

For $0<x \leq 1, s>1$ and $u \in N$

$$
\Phi(z, \quad s, \quad x, u)=\sum_{n=0}^{\infty} \frac{z^{n}}{\left(n^{u}+x\right)^{s}}
$$

Special cases of $H$-Lerch Zeta function are as follows:

The Lerch Zeta function

$$
\Phi(z, s, x)=\sum_{n=0}^{\infty} \frac{z^{n}}{(n+x)^{s}}=\Phi(z, s, x, 1)
$$

The Riemann Zeta Function, 
$\zeta(s)=\sum_{n=1}^{\infty} \frac{1}{n^{s}}=\Phi(1, s, 1,1)$

The Hurwitz Zeta Function.

$\zeta(s, x)=\sum_{n=0}^{\infty} \frac{1}{(n+x)^{s}}=\Phi(1, s, x, 1)$

The alternating zeta function, Also it is known as Dirichlet eta function $\eta(s)$

$$
\eta(s)=\sum_{n=1}^{\infty} \frac{(-1)^{n-1}}{n^{s}}=\Phi(-1, s, 1,1)
$$

The Dirichlet beta function

$$
\beta(s)=\sum_{n=0}^{\infty} \frac{(-1)^{n}}{(2 n+1)^{s}}=2^{-s} \cdot \Phi\left(-1, s, \frac{1}{2}, 1\right)
$$

The Legendre Chi Function,

$$
\chi_{s}(z)=\sum_{n=0}^{\infty} \frac{(-1)^{2 n+1}}{(2 n+1)^{s}}=2^{-s} \cdot z \cdot \Phi\left(z^{2}, s, \frac{1}{2}, 1\right)
$$

The Polylogarithm Function,

$$
L i_{k}(z)=\sum_{n=1}^{\infty} \frac{z^{n}}{n^{k}}=z \cdot \Phi(z, k, 1,1)
$$

Moreover, from Pedro's result in [6] the polylogarithm function given by the integral

$$
z \cdot \Phi(z, k, 1,1)=L i_{k}(z)=\sum_{n=1}^{\infty} \frac{z^{n}}{n^{k}}=\frac{z}{\Gamma(k+1)} \int_{0}^{1} \frac{\log ^{k}(1 / x)}{(1-z x)^{2}} d x
$$

Now, in next theorem we will check the convergence of H- Lerch zeta function.

\section{Theorem 12:}

The Generalized Lerch Zeta function

$$
\Phi(z, s, x, u)=\sum_{n=0}^{\infty} \frac{z^{n}}{\left(n^{u}+x\right)^{s}}
$$

where $z$ is a real number, $u$ is any positive integer, $s>1$ and $0<x \leq 1$

Then, the Generalized Lerch Zeta Function is convergent for $|z| \leq 1$ and divergent for $|z|>1$. 


\section{Proof}

To prove this result, first of we consider the general term of above series (42) is $a_{n}=\frac{z^{n}}{\left(n^{u}+x\right)^{s}}$.

Now, using the Ratio test for this series (42), we have $\lim _{n \rightarrow \infty} \frac{a_{n+1}}{a_{n}}=|z|$

Therefore, it is convergent for $|z|<1$ and divergent for $|z|>1$.

Now, for $z=1$ the series in (42) given by

$\Phi(1, s, x, u)=\sum_{n=0}^{\infty} \frac{1}{\left(n^{u}+x\right)^{s}}$

In addition, for all integers $n \geq 0$, we have $\frac{1}{\left(n^{u}+x\right)^{s}} \leq \frac{1}{(n+x)^{s}}$

So, $\quad \Phi(1, s, x, u)=\sum_{n=0}^{\infty} \frac{1}{\left(n^{u}+x\right)^{s}}=\mathrm{O}(\zeta(s, x))$

As $\zeta(s, x)$ is convergent for $s>1$, the series in (42) is convergent for $z=1$.

Therefore, it is obviously convergent when $z=-1$.

\section{Corollary 13}

For $u$ is any positive integer, $s>1$ and $0<x \leq 1$, we have

$$
\begin{aligned}
& \text { - } \Phi(1, s, x, u)+\Phi(-1, s, x, u)=\sum_{n=0}^{\infty} \frac{1}{\left((2 n)^{u}+x\right)^{s}} \\
& \text { - } \Phi(1, s, x, u)-\Phi(-1, s, x, u)=\sum_{n=0}^{\infty} \frac{1}{\left((2 n-1)^{u}+x\right)^{s}} \\
& \text { - } \Phi(z, s, x, u)=-\frac{1}{s} \frac{\partial}{\partial x} \Phi(z, s+1, x, u)
\end{aligned}
$$

Next, we apply the same strategy outlined above to the calculation of H- Lerch zeta function $\Phi(z, s, x, u)$ by using PMS.

\section{Theorem 14:}

A series for the H- Lerch zeta function, which is valid for $0<x \leq 1$ and $s>1$ given by 
$\Phi(z, \quad s, \quad x, u)=\frac{1}{x^{s}}+\sum_{m=0}^{\infty} \sum_{j=0}^{m}\left(\begin{array}{c}m \\ j\end{array}\right) \frac{\Gamma(m+s)}{\Gamma(s) \cdot m !} \frac{(-1)^{m+j} \lambda^{2(m-j)} x^{j} \cdot z^{n}}{\left(1+\lambda^{2}\right)^{m+s}} \zeta(u(s+j))$

for $\lambda^{2}>\frac{x-1}{2}$ and $x>0$.

\section{Proof:}

We can represent the series in (42) as

$$
\Phi(z, \quad s, \quad x, u)=\frac{1}{x^{s}}+\sum_{n=1}^{\infty} \frac{z^{n}}{\left(n^{u}+x\right)^{s}}
$$

We can rewrite the above series (45) as follows:

$\Phi(z, \quad s, \quad x, u)=\frac{1}{x^{s}}+\sum_{n=1}^{\infty} \frac{z^{n}}{n^{u s}} \frac{1}{\left(1+\lambda^{2}\right)^{s}} \frac{1}{(1+A(n))^{s}}$

where $A(n)=\frac{\frac{x}{n^{u}}-\lambda^{2}}{1+\lambda^{2}}$ and $\lambda$ is arbitrary constant introduced by hand.

Here, for all $n \geq 1$ the condition $|A(n)|=\left|\frac{\frac{x}{n^{u}}-\lambda^{2}}{1+\lambda^{2}}\right|<1$ provided that $\lambda^{2}>\frac{x-1}{2}$ and $x>0$.

Now, from the Binomial theorem we have

$$
\frac{1}{(1+A(n))^{s}}=\sum_{m=0}^{\infty} \frac{\Gamma(m+s)}{\Gamma(s) \cdot m !}(-A(n))^{m}
$$

By substituting the value of $A(n)$ in equation (47), we get

$$
\frac{1}{(1+A(n))^{s}}=\sum_{m=0}^{\infty} \frac{\Gamma(m+s)}{\Gamma(s) \cdot m !} \frac{(-1)^{m}}{\left(1+\lambda^{2}\right)^{m}}\left(\frac{x}{n^{u}}-\lambda^{2}\right)^{m}
$$

Again using binomial theorem for last term of the above equation (48), so we obtain

$$
\frac{1}{(1+A(n))^{s}}=\sum_{m=0}^{\infty} \sum_{j=0}^{m}\left(\begin{array}{c}
m \\
j
\end{array}\right) \frac{\Gamma(m+s)}{\Gamma(s) \cdot m !} \frac{(-1)^{m+j} \lambda^{2(m-j)}}{\left(1+\lambda^{2}\right)^{m}} \frac{x^{j}}{n^{u j}}
$$

Hence, from equations (46) and (49)

$$
\Phi(z, \quad s, \quad x, u)=\frac{1}{x^{s}}+\sum_{n=1}^{\infty} \sum_{m=0}^{\infty} \sum_{j=0}^{m}\left(\begin{array}{c}
m \\
j
\end{array}\right) \frac{\Gamma(m+s)}{\Gamma(s) \cdot m !} \frac{(-1)^{m+j} \lambda^{2(m-j)}}{\left(1+\lambda^{2}\right)^{m+s}} \frac{x^{j} \cdot z^{n}}{n^{u(s+j)}}
$$


As the summation runs over positive integers $n$, the above equation given by

$$
\Phi(z, \quad s, \quad x, u)=\frac{1}{x^{s}}+\sum_{m=0}^{\infty} \sum_{j=0}^{m}\left(\begin{array}{c}
m \\
j
\end{array}\right) \frac{\Gamma(m+s)}{\Gamma(s) \cdot m !} \frac{(-1)^{m+j} \lambda^{2(m-j)} x^{j} \cdot z^{n}}{\left(1+\lambda^{2}\right)^{m+s}} \zeta(u(s+j))
$$

This completes the proof.

After proving the main result, equation (44), we now discuss some properties of the new series in (44). We can easily see that the series in (44) is independent of the arbitrary parameter $\lambda$, although $\lambda$ appear in the expression of series in (44). This happens because the series in equation (42) is independent of arbitrary parameter $\lambda$ and it is easy to see that the new series in (44) converges to the series in (42) for $\lambda^{2}>\frac{x-1}{2}$ and $x>0$. This means the series in (44) describes a family of series, each corresponding to a different value of $\lambda$ and each series converging to the same series in (42).

Now, consider the partial sum of the series (44)

$$
\Phi^{N}(z, s, \quad x, u)=\frac{1}{x^{s}}+\sum_{m=0}^{N} \sum_{j=0}^{m}\left(\begin{array}{c}
m \\
j
\end{array}\right) \frac{\Gamma(m+s)}{\Gamma(s) \cdot m !} \frac{(-1)^{m+j} \lambda^{2(m-j)} x^{j} \cdot z^{n}}{\left(1+\lambda^{2}\right)^{m+s}} \zeta(u(s+j))
$$

It obtained by restricting the infinite sum to the first $N+1$ terms. Clearly, the partial sum $\Phi^{N}(z, s, x, u)$ depends upon the arbitrary parameter $\lambda$ as we neglecting infinite number of terms. Here, we can use this feature to our benefit and fix $\lambda$ therefore the convergence rate of the series is maximal. To accelerate the convergence of this series (42), apply the same strategy using PMS, which we apply to before series in this paper.

Now, we more generalize the H-Lerch Zeta function as G- Lerch zeta function as follows:

\section{Definition 15}

For any real number $z$ and positive integer $u, a$ and $0<x \leq 1$, the $G$-Lerch Zeta function is define by the series

$$
\Phi(z, s, x, u, a)=\sum_{n=0}^{\infty} \frac{z^{n+a}}{\left(n^{u}+x\right)^{s}}
$$

Next, we will see some special cases of $G$-Lerch Zeta function,

For $a=1$, the G-Lerch Zeta function is H-Lerch Zeta function,

$$
\Phi(z, s, x, u)=\sum_{n=0}^{\infty} \frac{z^{n}}{\left(n^{u}+x\right)^{s}}=\Phi(z, s, x, u, 1)
$$

Take, $u=a=1$ in (52), then the Lerch Zeta function,

$$
\Phi(z, s, x)=\sum_{n=0}^{\infty} \frac{z^{n}}{(n+x)^{s}}=\Phi(z, s, x, 1,1)
$$

If, we take $z=u=a=1$ in G-Lerch Zeta function then it co-inside with the Hurwitz Zeta Function. 
$\zeta(s, x)=\sum_{n=0}^{\infty} \frac{1}{(n+x)^{s}}=\Phi(1, s, x, 1,1)$

Moreover, when $x=z=u=a=1$, it becomes Riemann Zeta Function,

$\zeta(s)=\sum_{n=1}^{\infty} \frac{1}{n^{s}}=\Phi(1, s, 1,1,1)$

Next, the alternating zeta function, Also it is known as Dirichlet eta function $\eta(s)$

$\eta(s)=\sum_{n=1}^{\infty} \frac{(-1)^{n-1}}{n^{s}}=\Phi(-1, s, 1,1,1)$

The Dirichlet beta function

$\beta(s)=\sum_{n=0}^{\infty} \frac{(-1)^{n}}{(2 n+1)^{s}}=2^{-s} \cdot \Phi\left(-1, s, \frac{1}{2}, 1,1\right)$

The Legendre Chi Function,

$$
\chi_{s}(z)=\sum_{n=0}^{\infty} \frac{(-1)^{2 n+1}}{(2 n+1)^{s}}=2^{-s} \cdot z \cdot \Phi\left(z^{2}, s, \frac{1}{2}, 1,1\right)
$$

The Polylogarithm Function,

$$
L i_{k}(z)=\sum_{n=1}^{\infty} \frac{z^{n}}{n^{k}}=z \cdot \Phi(z, k, 1,1,1)
$$

From the results in (41)and (60) we get,

$$
\Phi(z, k, 1,1,1)=\Phi(z, k, 1,1)=\frac{1}{\Gamma(k+1)} \int_{0}^{1} \frac{\log ^{k}(1 / x)}{(1-z x)^{2}} d x
$$

\section{Theorem 16}

The G- Lerch Zeta function

$$
\Phi(z, s, x, u, a)=\sum_{n=0}^{\infty} \frac{z^{n+a}}{\left(n^{u}+x\right)^{s}}
$$

where $z$ is a real number, $u$ and $a$ are any positive integers, $s>1$ and $0<x \leq 1$.

Then, the G- Lerch Zeta Function is convergent for $|z| \leq 1$ and divergent for $|z|>1$.

\section{Proof}

To prove this result, first of we consider the general term of above series (62) is $a_{n}=\frac{z^{n+a}}{\left(n^{u}+x\right)^{s}}$. 
Now, using the Ratio test for this series (62), we have $\lim _{n \rightarrow \infty} \frac{a_{n+1}}{a_{n}}=|z|$

Therefore, the series in (62) is convergent for $|z|<1$ and divergent for $|z|>1$.

Now, for $z=1$ the series in (62) given by $\Phi(1, s, x, u, a)=\sum_{n=0}^{\infty} \frac{1}{\left(n^{u}+x\right)^{s}}$

And for all integers $n \geq 0 \frac{1}{\left(n^{u}+x\right)^{s}} \leq \frac{1}{(n+x)^{s}}$

Therefore, $\Phi(1, s, x, u)=\sum_{n=0}^{\infty} \frac{1}{\left(n^{u}+x\right)^{s}}=\mathrm{O}(\zeta(s, x))$

As $\zeta(s, x)$ is convergent for $s>1$, the series in (62) is convergent when $z=1$.

In addition, we can easily see that the series in (62) is convergent for $z=-1$.

Now, we apply the same strategy to G-Lerch Zeta function as we apply to H- Lerch Zeta function, obtain its parametric representation as given following theorem, and accelerate its convergence by applying PMS.

\section{Theorem 17:}

A series for the G- Lerch zeta function, which is valid for $0<x \leq 1$ and $s>1$ given by

$$
\Phi(z, \quad s, \quad x, u, a)=\frac{1}{x^{s}}+\sum_{m=0}^{\infty} \sum_{j=0}^{m}\left(\begin{array}{c}
m \\
j
\end{array}\right) \frac{\Gamma(m+s)}{\Gamma(s) \cdot m !} \frac{(-1)^{m+j} \lambda^{2(m-j)} x^{j} \cdot z^{n+a}}{\left(1+\lambda^{2}\right)^{m+s}} \zeta(u(s+j))
$$

for $\lambda^{2}>\frac{x-1}{2}$ and $x>0$.

\section{REFERENCES}

1. Flajolet P. And Vardi I., Zeta Function Expansions of Classical Constants, Unpublished manuscript, 1996

2. Gradshteyn I. S. and Ryzhik I. M., Table of integral series and products, Fourth edition, Academic press, New York and London, 1965

3. Modi H.B. and Ranabhatt G.A., Generalized Alternating Series II, ActaCienciaIndica, 2009, Vol, XXXV M, No. 4, $1183-1191$.

4. Modi H.B. and Ranabhatt G.A., Generalized Alternating Series,Bulletin of the Allahabad Mathematical Society, 2005, Vol. 20,p.89-98.

5. Modi H.B. and Ranabhatt G.A., Generalization of a series, ActaCienciaIndica, 2005, Vol. XXXI M, Nos. 2, 361-364.”

6. Pedro Jodra, On a connection between the Polylogarithm function andthe Bass diffusion mode, Proc.R. Soc. A 2008 464, 3081 3088 .

7. Ranabhatt G.A., A problem related to alternating seriesand allied topics, Ph.D. Thesis-Department of Mathematics,Maharaja Krishnakumarsinhji Bhavnagar University(https://shodhganga.inflibnet.ac.in/handle/10603/8339?mode=full),December 2011. 
8. Stevenson P. M., Optimized Perturbation Theory, Phy. Rev. 1981, D23, 2916.

9. Stivastava H. M. and Cho J. I, Series Associated with the zetand Releted Functions, Kluwer Academic Publishers, Dordrecht, theNetherlands, 2002.

10. Vardi. I., Computational Recreation in Mathematica, Addison Wesley, 1991 